\title{
Un debate sobre la teoría del derecho y la filosofía de la acción. Introducción
}

\author{
A Debate about the Theory of Law and the \\ Philosophy of Action. Introduction
}

María Gabriela Scataglini*

Recepción y evaluación de propuesta: 03/12/2019

Aceptación: 23/12/2019

Recepción y aceptación final: 27/02/2021

\begin{abstract}
Resumen: En el trabajo de Verónica Rodríguez-Blanco presentado y debatido en este número se propone el siguiente vínculo entre la teoría del derecho y la teoría de la acción: el teórico del derecho debería partir de una teoría correcta de la acción humana, a saber, una que permita captar el "sentido y propósito" (point) de las acciones de los participantes de la práctica jurídica. Según la autora, la metodología hartiana del punto de vista interno es "inestable" porque no logra este objetivo. Tres son las críticas que enfrenta la autora: una que discute principalmente el alcance y objetivo de la teoría de Hart; otra que cuestiona que el punto de vista interno revista algún privilegio como metodología científica; y una tercera que discute centralmente cuál sería una teoría correcta de la acción humana. Por mi parte, teniendo en cuenta la distinción entre explicaciones normativas y causales, destaco que no toda explicación basada en reglas presupone la representación por parte del agente del propósito valioso de la práctica o institución.
\end{abstract}

Palabras clave: acción humana, seguimiento de reglas, explicaciones normativas/causales, no representación.

Doctora de la Universidad de Buenos Aires. Profesora adjunta (interina) de Teoría General del Derecho de la Universidad de Buenos Aires, Argentina.

Correo electrónico: g_scataglini@hotmail.com 


\begin{abstract}
In Verónica Rodríguez-Blanco's paper presented and discussed in this volume, she points out the following link between the theory of law and the theory of action: the legal theorist should have a starting point in a sound theory of human action, namely: that which allows to capture the point of the actions of the participants of legal practice. According to the author Hart's methodology of the internal point of view is "instable" because it does not achieve this goal. She faces three criticisms: one mainly discussing the scope and purpose of Hart's theory; another criticizing the internal point of view has some privilege as a scientific methodology, and a third one mainly discussing what would be a sound theory of human action. For me, having in mind the distinction between normative and causal explanations, I stress that not all explanation based on rules presuppose the agent representing the valuable purpose of the practice or institution.
\end{abstract}

Keywords: human action, rule-following, normative explanations/causal explanations, no representation.

\title{
1. El planteo de Rodríguez-Blanco**
}

En este número se presenta el trabajo de Verónica Rodríguez-Blanco "Rastreando las críticas de Finnis al punto de vista interno de Hart: inestabilidad y el 'sentido y propósito' de la acción humana en el derecho”, y un debate en torno al mismo a cargo de tres comentaristas, cuyas críticas la autora responde.

El tema principal gira en torno a qué es lo que necesita comprender el teórico del derecho para tener una aproximación adecuada al fenómeno jurídico (i. e. qué distinciones debe poder trazar; qué debe poder explicar). Estas no son preguntas nuevas. Lo novedoso e interesante del planteo de Rodríguez-Blanco es que propone que para poder responderlas se debe partir de una teoría de la acción humana que sea correcta.

\footnotetext{
** El trabajo de Verónica Rodríguez-Blanco aquí introducido y algunos de los comentarios en sus versiones preliminares fueron presentados en el Taller Especial "Diálogos con Verónica Rodríguez-Blanco sobre Teoría del Derecho y Filosofía de la Acción” realizado el 26 de septiembre 2019 en la Facultad de Derecho de la Universidad de Buenos Aires, organizado por la Asociación Argentina de Filosofía del Derecho.
} 
Un debate sobre la teoría del derecho y la filosofía de la acción. Introducción

La autora introduce así una dimensión que podría parecer extraña al debate sobre metodología en la teoría del derecho: qué es lo que hace inteligible nuestras acciones y las de los otros, qué les da sentido, cómo un tercero puede captar y explicar la acción que yo he realizado. Para Rodríguez-Blanco estas son las cuestiones que deberíamos abordar para poder tener una teoría del derecho que nos permita explicar aquello que — según sostendrá- la metodología del punto de vista interno de Hart no alcanza a iluminar.

En la primera parte de su artículo, la autora reseña la crítica que Finnis dirige a Hart cuando le reprocha que la explicación del derecho en términos del punto de vista interno de los participantes sería insuficiente o "inestable”, básicamente porque no permitiría distinguir entre casos centrales y casos defectuosos de derecho.

Para Rodríguez-Blanco hay un aspecto de esa crítica de Finnis que ha sido pasado por alto y que es en el cual ella va a profundizar, a saber: ¿qué significa que el punto de vista interno de Hart es "inestable"? La respuesta de Finnis es que no permite hacer distinciones que cualquier actora en el campo (por ejemplo, la "ciudadana respetuosa del derecho") consideraría relevantes y estaría interesada en hacer. ¿Cuáles serían esas distinciones? Aquellas por ejemplo entre una norma buena y otra no tan buena, entre una directiva justa y una directiva injusta, entre una decisión racional de un tribunal y una decisión irracional.

Rodríguez-Blanco coincide con Finnis en que para poder hacer esas distinciones debe tomarse el punto de vista del agente que actúa con racionalidad práctica. Pero afirma que esa crítica de Finnis a Hart puede ser reconducida en los siguientes términos: el punto de vista interno hartiano es "inestable" porque no puede ser usado para entender el "sentido y propósito" (point) de las acciones humanas. Con esta afirmación, Rodríguez-Blanco establece el vínculo entre la teoría del derecho y la teoría de la acción, del cual va a ocuparse.

Ahora bien, las teorías de la acción no son todas iguales. Para la autora la inestabilidad que afecta al punto de vista interno hartiano obedece a que parte de una mirada sobre la acción humana que es inadecuada. Rodríguez-Blanco afirma que para explicar las acciones humanas se debe adoptar un enfoque basado en el resultado valioso o en las propiedades benéficas de la acción o práctica social en cuestión; esas propiedades iluminan el 
sentido y propósito de la acción del agente que participa en la práctica y la hacen inteligible. El análisis que propone la autora sigue las ideas de Elizabeth Anscombe; se trata de una explicación que mira a la exterioridad (outward-looking), al resultado de la acción. En contraposición se encuentran quienes asumiendo un enfoque introspectivo (inward-looking) - que mira a lo que sucede en la mente del sujeto- explican la acción humana tomando a los estados mentales como su causa (paradigmáticamente, D. Davidson). En cambio, en su célebre Intention, Anscombe afirma que la adecuada descripción de una acción resulta de la institución, juego del lenguaje o práctica social de la que participa el agente, y su estado mental no opera como causa de la acción: los movimientos corporales y las razones de la persona que actúa constituyen una unidad y así deben ser apreciadas. Para ejemplificarlo en pocas palabras: dado que participamos de la institución o práctica social del saludo, no describiríamos correctamente la acción de alguien que ve pasar a un amigo como "levantó su mano y la movió de un lado a otro", sino como "saludó".

En la segunda parte de su trabajo, Rodríguez-Blanco se dedica a criticar minuciosamente los enfoques causales de la acción y a dar argumentos a favor de una teoría de las acciones que explique su sentido y propósito a partir de las propiedades benéficas de la práctica social o juego del lenguaje. Saber qué juego del lenguaje está jugando el agente ilumina su "intención o elección”, y esa elección implica una deliberación en la cual quien actúa pondera los fines últimos del juego en cuestión, los elige y en razón de ello hace lo que hace.

Concluye que el déficit de la explicación causalista es que no puede garantizar que el vínculo causal entre una razón y la correspondiente acción sea el "correcto": y aquí "correcto" alude a que el teórico causalista no puede captar (ni por lo tanto asegurar) si el agente comprende el sentido y propósito de la acción y si se compromete con ella con motivo de ese sentido y propósito, o si meramente ha actuado coincidiendo con la regla por casualidad.

En la tercera parte, la autora retoma la teoría del derecho para mostrar por qué la metodología hartiana del punto de vista interno es, según ha dicho, "inestable". Para Rodríguez-Blanco, Hart asumiría un enfoque introspectivo (inward-looking) de la acción, tomando los estados mentales 
Un debate sobre la teoría del derecho y la filosofía de la acción. Introducción

- por ejemplo las creencias de los jueces y funcionarios aceptantes de la regla de reconocimiento- como la causa de sus acciones. Afirma la autora:

La idea de Hart de que aceptamos las normas jurídicas desde una perspectiva interna presupone una aproximación introspectiva a la acción como opuesta a una aproximación que mira hacia la exterioridad. Esta última examina las acciones intencionales como una serie de acciones que están justificadas en términos de otras acciones y en vista del propósito o finalidad de la acción intencional en tanto que una característica benéfica, por ejemplo, encender la tetera para hervir el agua, para hacer té porque es placentero beber té. Lo primero examina los estados mentales que racionalizan las acciones. Sin embargo, en el ámbito ontológico, se argumenta que esos estados mentales causan las acciones. Los estados mentales están compuestos por la creencia y la actitud proactiva hacia la acción (Rodríguez-Blanco, 2021, sección 3).

Para resumir, me animo a reconstruir la estructura argumental del trabajo de Rodríguez-Blanco así: i) una teoría del derecho adecuada es aquella que, tomando el punto de vista del agente que actúa con racionalidad práctica, permite al teórico realizar las mismas distinciones que son significativas y relevantes para el/la participante de la práctica del derecho; ii) una teoría de la acción adecuada es aquella que capta el "sentido y propósito" de las acciones de los agentes; y iii) la teoría hartiana del punto de vista interno no permite captar el "sentido y propósito" de las acciones, y consecuentemente no permite realizar aquellas distinciones significativas y relevantes para el/la participante de la práctica jurídica, por lo tanto, la de Hart no es una teoría del derecho adecuada.

No me toca discutir a mí sino a los comentaristas cuestiones tan interesantes como las aquí reseñadas. Sin embargo, hacia el final de esta introducción me permitiré realizar alguna reflexión general sobre el trabajo de Rodríguez-Blanco. 


\section{La cuestión metodológica: los comentarios de Scavuzzo y Guevara Arroyo}

Natalia Scavuzzo y Alejandro Guevara Arroyo abordan principalmente la cuestión metodológica, aunque dirigen su crítica a la autora desde posiciones diametralmente opuestas.

Articulando ideas del segundo Wittgenstein y de Peter Winch, Scavuzzo destaca que los significados de los enunciados realizados por los participantes están constituidos por el uso que ellos hacen de dichos enunciados en el marco de una práctica (Scavuzzo, 2021, sección 3).

Asumiendo cierto contextualismo (quizás más próximo a Winch) afirma que la comprensión de prácticas sociales requiere identificar el sentido que estas tienen para los agentes que las conforman y que este sentido es atribuido por las reglas pertenecientes a dichas prácticas. Ahora bien, en contraposición a Rodríguez-Blanco, Scavuzzo entiende que el punto de vista interno resulta idóneo a efectos de esa comprensión, mientras que el punto de vista de la racionalidad práctica no.

¿Por qué el punto de vista de quien actúa con racionalidad práctica no permitiría la comprensión de la práctica social de la que se pretende dar cuenta? Según Scavuzzo porque el punto de vista práctico parece ser simplemente el punto de vista de cualquier persona que se pregunta cómo actuar o que quiere entender cómo actúan los demás, pero nada de esto depende de (no está arraigado o anclado a —agregaría yo si no la interpreto mal-) una práctica social concreta.

El punto de vista de quien actúa con racionalidad práctica sería igual para todas las personas, sostiene Scavuzzo, independientemente de la práctica de que se trate. Así, la reducción del punto de vista interno - al cual Scavuzzo entiende como contextual - al punto de vista práctico haría perder todo sentido a la distinción entre punto de vista interno y externo planteada por Hart.

A su vez, cuestionando las ideas de Rodríguez-Blanco relativas a los fines benéficos de la práctica presuntamente perseguidos por los participantes, Scavuzzo señala que estos usan las reglas que sirven a los propósitos contingentes esa práctica, pero que ello es independiente de la bondad de esta. En última instancia, Rodríguez-Blanco habría malinterpretado el objetivo y la utilidad de la empresa hartiana. 
Un debate sobre la teoría del derecho y la filosofía de la acción. Introducción

En las antípodas de Scavuzzo, Alejandro Guevara Arroyo aborda también la cuestión metodológica de manera crítica al planteo de Rodríguez-Blanco. Él discrepa con el punto en que las autoras recién mencionadas coinciden: que la autocomprensión de las personas de la práctica en la que participan constituya un elemento fundamental para una teoría adecuada de dicha práctica.

Y aquí Guevara se opone tanto a la metodología del punto de vista interno como a la del punto de vista de quien actúa con racionalidad práctica. Puntualmente, frente a Rodríguez- Blanco, lo que Guevara cuestiona es qué razones epistemológicamente relevantes hay para asumir que el concepto construido adoptando el punto de vista de quien actúa con racionalidad práctica muestre - o nos permita dar con — la naturaleza del derecho o lo que el derecho es (Guevara Arroyo, 2021, Introducción).

De hecho, para el autor, parecería que la metodología del punto de vista de las personas que participan de una práctica no reviste ningún privilegio a los efectos de explicar sus acciones o el fenómeno del que estas forman parte.

Luego de algunas consideraciones científicas generales, señala que en ciencia hay diversos métodos de construcción de conceptos clasificatorios, puntualizando que estos deben cumplir condiciones de adecuación formales y materiales. Estas últimas - las condiciones materiales - requieren que la clasificación o partición que establece el concepto en cuestión no puede ser arbitraria, sino estar sometida a metodologías científicas de evaluación y sustentación, que las hacen preferibles como formas de averiguar cómo es el mundo y sus partes.

Bien, respecto del fenómeno jurídico en particular, esto es, para explicar qué es y cómo es el derecho, Guevara entiende que se deberían dar razones de por qué el punto de vista del agente que actúa con racionalidad práctica tal como lo entiende Rodríguez-Blanco (esto es a efectos de captar el sentido y propósito de sus acciones) debería ser el asumido por quien se dedica a la teoría del derecho.

Dado que no encuentra justificación suficiente en el trabajo de Rodríguez-Blanco, afirma que la metodología práctico-explicativa defendida por la autora no nos garantiza alcanzar un concepto adecuado de la naturaleza del derecho. 


\section{Monti: una discusión sobre teorías de la acción}

Monti realiza algunas precisiones respecto de cómo él —a diferencia de Rodríguez-Blanco- entiende la teoría hartiana del punto de vista interno, y señala que la crítica de "inestabilidad" que formula Finnis no estaría relacionada con la que realiza la autora (Monti, 2021, sección 2).

Más allá de esta cuestión, el núcleo del comentario de Monti consiste en discutir lo relativo a qué teoría de la acción sería la adecuada. Se detiene a analizar cada uno de los argumentos dirigidos por Rodríguez-Blanco contra los enfoques causales (el argumento de las cadenas causales desviadas, el de la memoria y el de la transparencia) y argumenta que ninguno de ellos logra su objetivo. La posición de Monti — minuciosamente defendida mediante ejemplos- es que el enfoque causal y el que propone Rodríguez-Blanco constituyen explicaciones complementarias, no excluyentes; ya que para toda acción intencional, es posible "traducir" su explicación desde el punto de vista deliberativo en términos de las características que hacen que sea valioso o bueno realizarla, a una explicación en términos de los estados mentales del agente.

Respecto de Hart, Monti coincide con la autora en que el autor inglés adopta un enfoque inward-looking de la acción en la medida que reconstruye el punto de vista interno en términos de los estados mentales (aceptación, creencias) del agente. Sin embargo, no admite que ello implique aceptar que Hart se comprometa con una teoría causal de la acción. Por lo tanto, para Monti, la explicación hartiana del punto de vista interno no resultaría vulnerable a la crítica "anticausal” de Rodríguez Blanco.

Por último, Monti da su propia interpretación de los argumentos razianos sobre el agente que actúa desde un punto de vista no comprometido, y discute críticamente la idea de que las normas jurídicas sean obligatorias o constituyan una razón para la acción porque es bueno o valioso actuar como estas requieren. Punto este que resulta aclarado en las réplicas. 
Un debate sobre la teoría del derecho y la filosofía de la acción. Introducción

\section{Algunas reflexiones sobre el planteo general de Rodríguez-Blanco}

Por último, permítaseme esbozar algunas consideraciones propias acerca del planteo general de Rodríguez-Blanco.

A grandes rasgos puede sostenerse que en filosofía hay dos tipos de explicaciones: las normativas, en términos de reglas, y las causales, en términos de eventos.

En el marco más específico de una teoría de la acción humana, una explicación normativa busca que sea posible decir si una acción es correcta o incorrecta con referencia a una regla: por ejemplo, si se la invoca como justificación de la acción, si la acción se reconoce como una instancia de la regla, cómo se debe actuar conforme a la regla.

En cambio, en una explicación causal se busca describir o identificar la acción a partir de qué es lo que la produjo. Para ello se suele apelar a lo que sucede en la mente del agente. Se trata de una explicación en términos de eventos (en el caso, eventos mentales) que causan - y a su vez explican — la acción del sujeto actuante. ${ }^{1}$

Parece claro que, en esta división general, Rodríguez-Blanco estaría a favor de una explicación normativa de la acción humana, y no de una causal.

Ahora bien, en su trabajo se alude a una distinción más específica, propia de las teorías de la acción: aquella ya antes reseñada entre un enfoque que mira a la exterioridad (outward-looking) entendiendo a esa "exterioridad" como el resultado o las propiedades valiosas (o el logos) de la práctica social, y un enfoque introspectivo (inward-looking). Y la autora no duda en alinear a los enfoques outward looking con aquellos que apelan a la racio-

1 Por supuesto que una explicación normativa no niega - y no tiene por qué hacerloque los eventos mentales tengan lugar, que ocurran en la mente de los sujetos. Pero lo que la caracteriza como normativa es que tales estados mentales no dan cuenta de lo que se busca explicar: a saber, si una acción es correcta en el marco de una práctica social determinada. "A neural or mental state on the other hand could only causally determine a consequence, not normatively or logically. It could not determine whether what is done is right, nor could it suffice to render behaviour an instance of rule-following at all. The linguist fails to grasp the categorial distinction between a state and an ability (power or potentiality). Consequently he misconstrues the conceptual relationship between an ability and its manifestations" (Baker, G. \& Hacker, P., 1984, p. 283). 
nalidad práctica del agente, por un lado, y a los enfoques introspectivos (inward-looking) con las explicaciones causales, por el otro. ${ }^{2}$

Bien, si se retoma lo que afirmé supra: que las explicaciones normativas son en términos de reglas y las causales en términos de eventos (i.e. estados mentales), se advierte que, dicho esquemáticamente, la contraposición relevante entre ambos tipos de abordaje filosófico se da entre estos dos elementos explicativos: la acción conforme a reglas, por un lado, y los estados mentales, por el otro.

Pero la alternativa presentada Rodríguez-Blanco apunta a una contraposición distinta: el primer elemento - i. e. la acción conforme a reglasaparece reemplazado por el logos o propósito de la acción. Este movimiento no es inocuo. Porque puestas las cosas en los términos de Rodríguez-Blanco parece que si queremos explicar las acciones humanas, nos enfrentamos a la siguiente alternativa: o bien tomamos a los eventos mentales como su causa, o bien consideramos al logos o propósito de la práctica como lo que explica la acción del agente. No estoy convencida de que esa disyuntiva esté planteada correctamente.

Una cosa es una explicación normativa, como opuesta a una causal, y otra cosa es una explicación como la que nos presenta Rodríguez-Blanco, y que me atrevo a caracterizar como "teleológica" (quizás esta sea una especie del género de las explicaciones normativas). A mi modo de ver, no toda explicación no causal (es decir, normativa) ha de ser teleológica. En particular, no toda explicación basada en reglas requiere apelar a la representación por parte del agente del fin valioso de la práctica en cuestión y a su elección, previa deliberación. ${ }^{3}$

Creo que es posible un enfoque normativo (no causal) de la acción humana basado en las habilidades del agente para seguir reglas en el marco de una práctica social, sin más. La ausencia de un elemento repre-

2 Esto resulta dudoso, puesto que podría haber enfoques introspectivos no necesariamente causales, pero no me ocuparé de ello aquí.

3 Por supuesto que nada obsta a que tengamos reflexiones críticas acerca de los valores de las instituciones o prácticas sociales en las que participamos, pero lo que estoy defendiendo es que esa reflexión crítica y consecuente elección del agente en vista de los propósitos de la acción no son conceptualmente necesarias para que su acción resulte inteligible para el observador. 
Un debate sobre la teoría del derecho y la filosofía de la acción. Introducción

sentacional y/o deliberativo no hace mella en el carácter práctico o intencional de la acción. ${ }^{4}$

Me explico: un agente que actúa intencionalmente en el marco de un juego del lenguaje o un contexto institucionalizado está siguiendo reglas. Ahora bien, la noción de "seguir una regla" no tiene por qué concebirse como requiriendo que el agente se represente una proposición discursiva, explícita y general - a modo de primera premisa de su razonamiento práctico- que justifique su acción. Podría, en su lugar, estar simplemente respondiendo al modo en que ha sido entrenado para seguir la regla. Ese entrenamiento se da en el marco de una costumbre o práctica social. ${ }^{5}$ En este sentido, el seguimiento de reglas puede ser "ciego" - en términos wittgensteinianos- en tanto no implica la realización de operaciones mentales abstractas previas a la acción; no se requiere una reflexión o representación de por medio. Una sabe qué hacer no por seguir una máxima, sino porque tiene la habilidad de responder de cierta manera distintiva al enfrentarse a las situaciones concretas.

La referencia a actuar "ciegamente" de ningún modo significa que ese actuar sea de tipo "estímulo-respuesta” o "irracional”. Piénsese en el jugador de ajedrez experto que juega rápido y eficientemente: en ocasiones no puede explicar en términos de proposiciones por qué actuó así o cómo hizo para hacerlo del modo correcto más que de una manera insuficiente o indeterminada. Pero esto no significa que su accionar no haya sido normativo, ni tampoco que no haya sido intencional. Por el contrario, está tan imbuido de las reglas que tiene un seguimiento ciego o no representacional de las mismas, y entonces ante la pregunta de qué hizo — si bien la entiende y le es potencialmente posible conceptualizar (al menos en alguna descripción) qué ha hecho-, no puede explicar, más que difusamente, el cómo ni el porqué. En síntesis, la incapacidad para explicar o el carácter opaco de su proceder, no constituyen un obstáculo para afirmar que actuó siguiendo

4 Es dable destacar que en sus réplicas -a diferencia de lo afirmado en el trabajo en discusiónRodríguez- Blanco parece aceptar esta última idea.

5 Como señala Patterson, la relevancia del entrenamiento no es causal, sino normativa: "What Wittgenstein is pointing to here is the connection between meaning and training (...) Wittgenstein maintains that the key to normativity lies in the ways in which we have been trained to respond to the sign or rule" (Patterson 2001, p. 342). 
reglas, ni para que su acción nos resulte inteligible como acción racional e intencional.

McDowell (2013) explica que nuestras acciones son racionales (él usa alternativamente "conceptuales") en el sentido de que son susceptibles tienen la potencialidad-de formar parte en la justificación de una acción, lo cual es inseparable de nuestra capacidad lingüística. Pero aclara que esto es así aun si la respuesta a por qué un agente actuó de cierta manera es indeterminada o no concluyente. Lo ilustra con el ejemplo "automático" de atrapar un disco volador: cuando un agente racional atrapa un disco volador está poniendo en práctica un concepto de una cosa que sabe hacer, digamos, atrapar esto; en el caso del experto no lo hace poniendo en práctica otros conceptos (si no lo entiendo mal, aquí McDowell se estaría refiriendo a "otras" cosas tales como por ejemplo "estirarme", "poner la vista en el objeto", o también "no debo dejar que caiga", "no quiero perder el juego" etc.). Pero lo que distingue el actuar de un agente racional, sea experto o no, del actuar de un perro que atrapa el disco es que el perro no pone en práctica ningún concepto práctico; en sentido relevante, no tiene ninguno. Afirmar que el agente racional, a diferencia del perro, pone en práctica un concepto a través de una acción es afirmar que su acción (bajo una especificación que captura el contenido del concepto práctico que está llevando a cabo) está dentro del alcance de su racionalidad práctica, aún si su respuesta a la pregunta de por qué atrapó el disco fuera tan insuficiente como: "Por ninguna razón en particular. Simplemente tuve ganas" $(2013$, p. 35$){ }^{6}$

6 La traducción es mía; en el original: “No particular reason. I just felt like it!". En la disputa teórica ahora ya conocida como el "debate McDowell-Dreyfus", el tema de si el actuar "sin razones" puede considerarse aún un actuar racional ha alcanzado matices muy finos. El debate tiene varias instancias, la última de ellas, conteniendo además contribuciones de otros autores, puede encontrarse en Schear (2013). Allí se presenta el contraste entre lo que sería una mirada estrictamente fenomenológica (representada por Dreyfus) y otra mirada de corte wittgensteiniano-ryleano (encarnada por McDowell). Simplificando en extremo, para Dreyfus (2013), cuando nos enfrentamos al entorno, por ejemplo, al desarrollar cualquier actividad (por caso jugar al ajedrez, atrapar un disco volador, pero también acciones cotidianas como qué distancia corporal mantener con otras personas en diversos contextos), se pone en juego una normatividad sin que medie racionalidad: quien actúa de manera experta, con maestría, lo hace absorto en la práctica, hay una suerte de enfrentarse (to cope) al entorno que no requiere de ningún tipo de mediación conceptual o representación. En cambio, McDowell sostiene que "capacities that are conceptual, capacities that belong to 
Un debate sobre la teoría del derecho y la filosofía de la acción. Introducción

Todo esto parecería contrastar con lo sostenido por Rodríguez-Blanco en el trabajo que aquí se discute. Ello en cuanto, para la autora, aun si en una primera respuesta el agente apelara de manera clara a la regla, esto sería insuficiente. Siguiendo la metodología del porqué de Anscombe, deberíamos seguir preguntándole por qué (por ejemplo, "por qué acepta la regla") hasta que su respuesta hiciera evidente su compromiso con los valores o propiedades benéficas (el logos) de la práctica en cuestión, y recién entonces habríamos logrado hacer inteligible su acción en términos de racionalidad práctica. Como he sugerido antes, esta parece más bien una explicación teleológica y no meramente una basada en reglas en el marco de una práctica social.

Unas últimas palabras en torno a Hart. Respecto de la distinción general que he planteado entre explicaciones normativas y causales, me parece claro que la metodología hartiana del punto de vista interno es de tipo normativa, y no causal. Por supuesto, la de Hart no es una teoría normativa del mismo modo que lo es la de Dworkin. Como positivista, Hart busca describir el derecho que es, y de hecho plantea la existencia de ambos puntos de vista, el interno y el externo. Pero lo que Hart sostiene es que, para describir adecuadamente la acción conforme a reglas - y no quedarse en la mera captación de regularidades y/o reacciones hostiles-, el teórico debe estar en condiciones de "ponerse en el lugar del participante" (Hart, 1997, p. 95). Se trata de "adoptar" el punto de vista interno; algo similar a lo que recientemente se ha llamado un "positivismo jurídico interno" (Redondo, 2018).

Ahora, situados en el punto de vista interno, si bien es cierto que Hart alude a cierto estado mental (la creencia) de los jueces y funcionarios, en el sentido de que se dice que ellos aceptan la regla de reconocimiento no por cualquier motivo o por motivos prudenciales, me parece altamente dudoso que ese elemento de aceptación funcione en su explicación como un evento mental que causa el accionar de los jueces.

Adviértase que, a diferencia de las explicaciones causales que apelan a instancias subjetivas como los estados mentales (aquí las creencias de los 
jueces), una metodología como la del punto de vista interno se centra en la práctica social: es en la arena intersubjetiva donde las reglas son obligatoriamente aplicadas, enseñadas, aprendidas y las acciones juzgadas como correctas o incorrectas respecto de ellas. En esa línea, cabe destacar que lo que requiere la teoría hartiana de la aceptación es que los jueces y funcionarios consideren a la regla de reconocimiento "como un criterio común $y$ público de decisiones judiciales correctas y no como algo que cada juez simplemente obedece por su cuenta" (1963, p. 144, destacados propios). Y más adelante reitera que si algunos jueces "actuaran 'por su cuenta' (...) y no apreciaran críticamente a aquellos colegas que no respetasen esta regla de reconocimiento, la característica unidad y continuidad del sistema jurídico habrían desaparecido" (Hart, 1963, p. 144, entrecomillado del original). Se trata de una explicación que apela a la intersubjetividad, a la idea de cómo se debe actuar en el marco de una institución o práctica social y no a la subjetividad de las creencias entendidas como estados mentales.

Creo que siguiendo esta línea pueden construirse argumentos para discrepar con la autora en torno a que el de Hart constituya un enfoque introspectivo, de tipo inward-looking. Pero ello no será en el marco de esta introducción, ya que es a los comentaristas a quienes toca debatir las sugerentes ideas de Rodríguez-Blanco.

\section{Bibliografía}

Baker, G. \& Hacker, P. (1984). Language, Sense and Nonsense. A Critical Investigation into Modern Theories of Language. Basil Blackwell, Oxford. Dreyfus, H. (2013). The myth of the pervasiveness of the mental, en Schear, J. Mind, Reason and Being-in.the-world. The McDowell-Dreyfus Debate (pp. 15-40). London- New York: Routledge.

Guevara Arroyo, A. (2021). Naturaleza y concepto de Derecho a los ojos de Rodríguez-Blanco: un análisis naturalista de su propuesta metodológica. Discusiones, 26(1).

Hart, H. L. A. (1963). El Concepto de Derecho. Buenos Aires: Abeledo Perrot. 
Hart, H. L. A. (1997). Post-scriptum. En La decisión judicial. El debate Hart-Dworkin. (Traducción al castellano: Magdalena Holguín), (pp. 89-141). Bogotá: Siglo del Hombre Editores.

McDowell, J. (2013). The myth of the mind as detached. En Schear, J. Mind, Reason and Being-in.the-world. The McDowell-Dreyfus Debate (pp. 41-58). London- New York: Routledge.

Monti, E. (2021). Rodríguez-Blanco sobre el carácter inestable del punto de vista interno. Discusiones, 26(1).

Patterson, D. (2001). Normativity and Objectivity in Law. En William \& Mary Law Review, 43(1), 325-363. Disponible en: http://scholarship. law.wm.edu/wmlr/vol43/iss1/9

Redondo, M. C. (2018). Positivismo jurídico “interno”. Liubliana: Klub Revus.

Rodríguez-Blanco, V. (2021). Rastreando las críticas de Finnis al punto de vista interno de Hart: inestabilidad y el "sentido y proposito" de la acción humana en el derecho Discusiones, 26(1).

Scavuzzo, N. (2021). Hart y el "sentido y propósito" del punto de vista interno. Discusiones, 26(1).

Schear, J. (2013). Mind, Reason and Being-in.the-world. The McDowellDreyfus Debate. London-New York: Routledge.

Wittgenstein, L. (1953 [1988]). Investigaciones Filosóficas. México: Instituto de Investigaciones Filosóficas de la UNAM. 